\title{
Oral carriage of candidiasis in patients with oral dental diseases: predisposing factors, species and their antifungal susceptibility patterns
}

\begin{abstract}
The aim of this study was to evaluate prevalence, frequency and predisposing factors as well as their antifungal susceptibility pattern of oral candidiasis (OC) in patients attending outpatient's dental clinic with various dental diseases. A total of 150 patients were enrolled in this study aged from 4 to 73 years attended outpatient dental clinic, at Zahra district Tripoli, Libya between May 20017 and May 2018. The purpose of this study was to evaluate the frequency of yeasts in the oral cavity, and to determine the main yeast species present, their correlation with various dental problems as well as other predisposing factors and there antifungal susceptibility patterns. One hundred and fifty patients were enrolled in this study. Patients were submitted to an odontological examination for the identification of dental caries and dental plaque, and other dental problems, as well as for yeast culture. Identification of all species was based on standard mycological methods and antifungal susceptibility test was performed by using disk diffusion susceptibility method and several antifungal drugs such as (fluconazole, amphotericin B, ketoconazole, econazole, itraconazole) were used. The frequency oral carriage of yeasts was $64 \%$ (96), with $78 \%$ aged from 20 to 60 years old. Caries, plaque, gingivitis periodontitis, and lower teeth are the most dental problem in which, more than $50 \%$ harbors candida species in their oral cavity. Diabetes, pregnancy and smoking, is among common predisposing factors. Candida albicans was the most prevalent species $(41.7 \%)$, C. glabrate $(27.1 \%)$ and C. dubliniensis $(11.5 \%)$. Susceptibility test show that Candida albicans was highly resistant to most azole antifungal and Candida dubliniensis was highly resistant to fluocytosine. Other candida species show variable susceptibility to various antifungal drugs.
\end{abstract}

Aim of study: The purpose of this study was to evaluate the frequency of yeasts in the oral cavity, and to determine the main yeast species present, their correlation with various dental problems as well as other predisposing factors and there antifungal susceptibility patterns. One hundred and fifty patients were enrolled in this study. Patients were submitted to an odontological examination for the identification of dental caries and dental plaque, and other dental problems, as well as for yeast culture. Identification of all species was based on standard mycological methods and antifungal susceptibility test was performed by using disk diffusion susceptibility method and several antifungal drugs such as (fluconazole, amphotericin B, ketoconazole, econazole, itraconazole) were used.

Material and methods: One hundred and fifty patients were enrolled in this study. Patients were submitted to an odontological examination for the identification of dental caries and dental plaque, and other dental problems, as well as for yeast culture. Identification of all species was based on standard mycological methods and antifungal susceptibility test was performed by using disk diffusion susceptibility method and several antifungal drugs such as (fluconazole, amphotericin B, ketoconazole, econazole, itraconazole) were used.

Results: The frequency oral carriage of yeasts was $64 \%$ (96), with $78 \%$ aged from 20 to 60 years old. Caries, plaque, gingivitis periodontitis, and lower teeth are the most dental problem in which, more than $50 \%$ harbors candida species in their oral cavity. Diabetes, pregnancy and smoking, is among common predisposing factors. Candida albicans was the most prevalent species $(41.7 \%)$, C. glabrate $(27.1 \%)$ and C. dubliniensis (11.5\%). Susceptibility test show that Candida albicans was highly resistant to most azole antifungal and Candida dubliniensis was highly resistant to fluocytosine. Other candida species show variable susceptibility to various antifungal drugs.

Conclusion: This finding indicated a correlation between the presence of caries and other dental diseases with yeasts carriage in the mouth. However, there was no clear cut correlation between yeasts carriage in healthy and health patients.
Volume 6 Issue 3 - 2018

\author{
Krema ZA,' Trfas EEM,' Ellabib MS,' Cogliati \\ $M^{2}$ \\ 'Department of Medical Microbiology and Immunology, Tripoli \\ University, Libya \\ ${ }^{2}$ Dip Scienze Biomediche per la Salute, Università degli Studi di \\ Milano, Italy
}

Correspondence: Mohamed Ellabib, Department of Medical Microbiology and Immunology, Faculty of Medicine, Tripoli University, P.O. Box 13497, Tripoli, Libya, Tel 02-1821-5857-6I9, Email ellabib@mail.com

Received: June 04, 2018 | Published: June 13, 2018

Keywords: candida species, gingivitis, prosthesis, diabetes 


\section{Introduction}

Different species of yeasts can colonize the oral mucosa under well-balanced ecosystem conditions. The primary etiological agent of oral candidiasis is the yeast $C$. albicans; however, other species that cause disease less commonly include $C$. tropicalis, $C$. glabrata, $C$. krusei, C parapsilosis, C. guilliermondii, and C. Dubliniens is. ${ }^{1}$

However, alterations in this equilibrium may transform these microorganisms into infectious agents, expressing virulence factors and invading tissues. ${ }^{2}$ The incidence of fungal infections has increased in recent years in many countries, which has stimulated scientific studies. ${ }^{1}$ In relation to oral infections, such as stomatitis, candida has been found in different situations such as root canal infections, especially in the root canals of obdurate teeth in which treatment has failed. C. albicans has been associated with cases of persistent root canal infections, because this yeast can be resistant to some intracanal medications. $^{3}$

Dental caries is a chronic and multifactorial disease that, although avoidable, still represents an important problem in public health, since it affects approximately $90 \%$ of the population, mainly children and adolescents, compromising their quality of life and development. ${ }^{4}$ The development of caries depends on the interaction of multi factors relating to the host, especially a diet rich in fermentable carbohydrates, and the presence of Cariogenic microorganisms ${ }^{5}$ Microbial process resulting from a nonspecific accumulation of acid-producing microorganisms on teeth; it should be interesting to confirm the possible involvement of other microorganisms in their formation. Classically, the microorganisms involved in the genesis and development of caries are bacteria such as Streptococcus mutans and other cocci and rods. ${ }^{6}$ Furthermore there is evidence of the involvement of C. albicans in the etiology of dental caries. ${ }^{7}$ Yeast cells are certainly acidogenic microorganisms, but the primary caries process has not yet been linked to the presence of yeast cells. Therefore, the aim of this study was to evaluate the frequency of yeasts in the oral cavity, determine the main species present, and assess the possibility of a correlation between the presence of yeasts with caries and dental plaque as well as other predisposing factors and their antifungal susceptibility patterns.

\section{Materials and methods}

\section{Patients oral rinse study sample}

One Hundred fifty asymptomatic individuals patients with dental oral disease referred to the dental clinics in ZAHRA City were enrolled in this study. Oral mouth rinse samples were taken as well as sample from infected teeth when involved. A patient's information sheet (Questionnaire survey) was used included patient information such as age, gender, oral condition, gingivitis, periodontitis, caries, smoking and any other predisposing factors.

\section{Samples collections and processing}

Samples were obtained by an oral rinse in which the mouth was washed for 30 second with $10 \mathrm{~mL}$ of sterile water. The mouthwash liquid was deposited in conical tubes and stored in an insulated container until microbiological processing. Later, this suspension was washed three times in sterilized $0.1 \mathrm{M}$ phosphate-buffered saline $\mathrm{pH}$ 7.4 (PBS) by centrifugation 3.000rpm, the pellet was resuspended in $1 \mathrm{~mL}$ of PBS, and $10 \mu \mathrm{L}$ aliquots were inoculated with a bacteriological loop onto the surface of Sabouraud's dextrose agar with antibiotic
(Oxoid Ltd). ${ }^{8}$ Plates were incubated at $30^{\circ} \mathrm{C}$ for 24 to $72 \mathrm{~h}$. Yeasts were identified by germ-tube production, micromorphology, and chlamydospores production on Tween 80-corneal agar. All isolates with germ tube test positive and chlamydospores positive on corn meal agar plus tween 80 were identified as $C$. albicans or $C$. dubliniensis. Sunflower seed agar and xylose hypertonic media ${ }^{9,10}$ was used to differentiate $C$. albicans from $C$. dubliniensis. Non candida albicans species were identified by API 20C AUX (bioMérieux's), Sample from involved teeth was suspended in $1 \mathrm{ml}$ of sterile normal saline, vortexes for 1 minute and $0.1 \mathrm{ml}$ subsequently inoculating on an Sabouraud's dextrose agar plus antibiotics (SDA) when required. ${ }^{11}$

\section{Antifungal susceptibility testing}

Five distinct colonies of approximately one $\mathrm{mm}$ from each 24 hours old culture grew on Sabouraud Dextrose Agar incubated at $35 \pm 2{ }^{\circ} \mathrm{C}$. Colonies were suspended in $5 \mathrm{~mL}$ of sterile $0.85 \%$ Saline. The resulting suspension was adjusted to the turbidity to yield $1 \times 10^{6}-5 \times 10^{6}$ cells/ $\mathrm{mL}$ (i.e., $0.5 \mathrm{McF}$ arland standard). A sterile cotton swab moistened with the inoculums suspension was used to apply to a $90 \mathrm{~mm}$ diameter plate containing Mueller-Hinton agar supplemented with $2 \%$ glucose and $0.5 \mu \mathrm{g} / \mathrm{mL}$ methylene blue (GM-MH agar medium) ${ }^{12,13}$ The plates were allowed to dry for 5-15 minutes before disks were placed in the center of the agar. The following antifungal discs was purchased from Liofilchem, Italy were used are Amphotericin B $(20 \mu \mathrm{g})$, Caspofungin $(5 \mu \mathrm{g})$, Clotrimazole $(50 \mu \mathrm{g})$, Econazole $(10 \mu \mathrm{g})$, Fluconazole $(25 \mu \mathrm{g}$ and $100 \mu \mathrm{g})$, Flucytosine $(1 \mu \mathrm{g})$, Itraconazole ( $50 \mu \mathrm{g})$, Ketoconazole $(10 \mu \mathrm{g})$, Miconazole $(10 \mu \mathrm{g})$, Nystatin (10 $100 \mathrm{IU})$, Posaconazole $(5 \mu \mathrm{g})$, Voriconazole $(1 \mu \mathrm{g})$. The plates were incubated for 18-24 hours at $37 \pm 2^{\circ} \mathrm{C}$ and the slowly growing isolates were again read after 48 hours incubation. Zone sizes interpreted according to CLSI document criteria for susceptible and resistant. ${ }^{14}$ Quality control: Quality control procedures were performed as per CLSI guidelines ${ }^{13}$ using C. albicans ATCC 90028 and C. parapsilosis ATCC 22019 strains, as quality control strains for fluconazole and voriconazole disk diffusion testing.

\section{Results}

Oral rinses sample were obtained from 150 patients, $54.7 \%(\mathrm{n}=82)$ females and $45.3 \%(n=68)$ males, most patients were between 20 and 60 years old with over $50 \%$ culture positive for yeasts. Female was more colonized by yeast than male and high yeast colonization was also found among patient age from 20 to 40 years old, (Table 1).

Table I Distribution of oral cavity cultures positive for yeasts according to gender, age, dental problem and predisposing factors

\begin{tabular}{llll}
\hline $\begin{array}{l}\text { Variable } \\
\text { analysis }\end{array}$ & $\begin{array}{l}\text { Total } \\
\text { number }\end{array}$ & $\begin{array}{l}\text { Positivie } \\
\text { culture }\end{array}$ & $\%$ \\
\hline $\begin{array}{l}\text { Oral cavity sample } \\
\text { Sex }\end{array}$ & 150 & 96 & 64 \\
Female & 82 & 55 & 7.1 \\
Male & 68 & 41 & 60.3 \\
Total & 150 & 96 & 64 \\
Age distribution & & 5 & 71.4 \\
$\leq$ than 20 & 7 & 40 & 72.7 \\
$\leq$ than 40 & 55 & 38 & 55.1 \\
\hline than 60 & 69 & 13 & 68.4 \\
\hline$>60$ & 19 & & \\
\hline
\end{tabular}


Table continuded

\begin{tabular}{llll}
$\begin{array}{l}\text { Variable } \\
\text { analysis }\end{array}$ & $\begin{array}{l}\text { Total } \\
\text { number }\end{array}$ & $\begin{array}{l}\text { Positivie } \\
\text { culture }\end{array}$ & $\%$ \\
\hline Total & 150 & 96 & 64 \\
Pregnant & 4 & 4 & 100 \\
Diabetes mellitus & 5 & 4 & 80 \\
PF only & 15 & 11 & 73.3 \\
Periodontitis & 11 & 8 & 72.7 \\
Dental caries & 18 & 12 & 66.7 \\
DC+PF & 65 & 43 & 66.2 \\
Gingivitis & 27 & 17 & 63 \\
DC only & 62 & 38 & 61.3 \\
NAD and No PF & 8 & 4 & 50 \\
Smoking & 5 & 2 & 40 \\
\hline
\end{tabular}

Out of 150 patients evaluated in this study $96(64 \%)$ patients were identified as oral candida carriers. C. albicans was identified in $(41.7 \%)$ of the oral rinse and $(36.4 \%)$ from teeth samples, being the predominant Candida species. Additionally, C. glabrate comprised (27.1\% and $27.3 \%$ respectively) of the isolates, followed by $C$. dubliniensis (11.5\% and 24.2 respectively). Detailed of yeast isolated from both oral rinse and teeth site are summarized in Table 2.

The antifungal susceptibility test as determined by the disc diffusion method for isolates from both oral rinse and teeth site are presented in Table 3. All Candida isolates tested were susceptible to amphotericin B, nystatin and Caspofungin. All C. albicans were highly resistant to fluconazole, Itraconazole, Posaconazole and voriconazole. C. dubliniensis, C. glabrate and other candida species isolates were more susceptible. All isolates including $C$. albicans were more susceptible to Clotrimazole, Econazole, Miconazole and ketoconazole. All isolates except $C$. guillermondii were determined as highly resistant to fluocytosine.

NAD (No Apparent Disease), DC (Dental compliant), PF (predisposing factor)

Carries gingivitis, dental plaque is most common dental compliments associated yeast carriage and lower teeth were more involved than upper teeth (73 and 18 respectively), however positive culture was higher among patients from upper teeth problem $(77.8 \%$ and $60.3 \%$ ). Cultures for yeast were positive in all cases even among patient with Non Apparent Disease (NAD) or non-diseased teeth. However positive culture for yeasts was higher in patient with dental compliant 38 out 62 positive culture $(61.3 \%)$, in contrast to patient with NAD and non-predisposing factors 4 out of $8(50 \%)$, as shown in Table 1. High frequency of positive culture for yeast in patient with predisposing factors than patient has non predisposing factors was also seen, in which out of 11 positive culture out of $15(73.3 \%)$ have at least has one predisposing factor Pregnancy and diabetes are the most common predisposing factor yield positive culture for yeast comparing to patient with any other risk factors. The lowest rate carriage 4 out $8(50 \%)$ positive cultures in our study was only observed among patient with free of both dental complaint and any predisposing factor (Table 1). Detailed culture positive for yeast of variable analysis are shown in Table 1 . The frequency positive of culture in patient with different risk factors is presented in Figure 1, which indicated that dental compliant and predisposing factors are major cause in oral carriage and colonization with yeast in our study.

Table 2 Type and frequency of yeast species isolated from oral rinse and teeth site specimens

\begin{tabular}{|c|c|c|c|c|c|}
\hline $\begin{array}{l}\text { Oral rinse } \\
\text { species }\end{array}$ & $\begin{array}{l}\text { Total } \\
\text { no }\end{array}$ & $\%$ & Site species & $\begin{array}{l}\text { Total } \\
\text { number }\end{array}$ & $\%$ \\
\hline Candida albicans & 40 & 41.7 & $\begin{array}{l}\text { Candida } \\
\text { albicans }\end{array}$ & 12 & 36.4 \\
\hline Candida glabrate & 26 & 27.1 & $\begin{array}{l}\text { Candida } \\
\text { glabrate }\end{array}$ & 9 & 27.3 \\
\hline $\begin{array}{l}\text { Candida } \\
\text { dubliniensis }\end{array}$ & II & 11.5 & $\begin{array}{l}\text { candida } \\
\text { dubliniensis }\end{array}$ & 8 & 24.2 \\
\hline Candida famata & 6 & 6.3 & $\begin{array}{l}\text { Cryptococcus } \\
\text { laurentii }\end{array}$ & 2 & 6.1 \\
\hline $\begin{array}{l}\text { Cryptococcus } \\
\text { humicolus }\end{array}$ & 4 & 4.2 & $\begin{array}{l}\text { Candida } \\
\text { famata }\end{array}$ & I & 3 \\
\hline $\begin{array}{l}\text { Cryptococcus } \\
\text { laurentii }\end{array}$ & 4 & 4.2 & $\begin{array}{l}\text { Cryptococccus } \\
\text { humicolus }\end{array}$ & I & 3 \\
\hline $\begin{array}{l}\text { Candida } \\
\text { guillermondii }\end{array}$ & 2 & 2.1 & & & \\
\hline $\begin{array}{l}\text { Trichosporoum } \\
\text { mucoides }\end{array}$ & 1 & I & & & \\
\hline $\begin{array}{l}\text { Saccharomyces } \\
\text { cereviseae }\end{array}$ & 1 & I & & & \\
\hline $\begin{array}{l}\text { Rhodotorula } \\
\text { minuta }\end{array}$ & 1 & I & & & \\
\hline Total & $96 / 150$ & & Total & $33 / 91$ & \\
\hline
\end{tabular}

Table 3 Antifungal susceptibility profile of Candida isolates by disk diffusion technique

\begin{tabular}{|c|c|c|c|c|c|c|c|c|c|c|c|c|c|}
\hline \multirow{2}{*}{$\begin{array}{l}\text { Oral einse species } \\
\text { Species }\end{array}$} & \multicolumn{13}{|c|}{$\begin{array}{l}\% \text { of } \\
\text { susceptibility }\end{array}$} \\
\hline & AMB & FLU25 & FLII 00 & POSS & NYIO & CLO50 & ITC50 & AFYI & VOI & CAS5 & KCAIO & MCLIO & ECNIO \\
\hline Candida albicans & 100 & 26.3 & 26.3 & 23.7 & 100 & 100 & 26.3 & 5.3 & 26.3 & 100 & 63.2 & 100 & 100 \\
\hline Candida glabrata & 100 & 80.8 & 80.8 & 80.8 & 100 & 100 & 76.9 & 7.7 & 76.9 & 100 & 80.5 & 100 & 100 \\
\hline Candida dubliniensis & 100 & 100 & 100 & 100 & 100 & 100 & 100 & 9.1 & 90.9 & 100 & 100 & 100 & 100 \\
\hline Candida famata & 100 & 83.3 & 83.3 & 66.7 & 100 & 100 & 83.3 & 0 & 83.3 & 100 & 100 & 100 & 100 \\
\hline Candida guillermondii & 100 & 100 & 100 & 100 & 100 & 100 & 100 & 50 & 100 & 100 & 100 & 100 & 100 \\
\hline Other yeast & 100 & 81.8 & 81.8 & 81.7 & 100 & 100 & 81.8 & 18.2 & 81.8 & 100 & 100 & 100 & 100 \\
\hline \multicolumn{14}{|l|}{ Teeth site species } \\
\hline Candida albicans & 100 & 36.4 & 36.4 & 36.4 & 100 & 100 & 27.3 & 27.3 & 36.4 & 100 & 72.7 & 100 & 100 \\
\hline Candida dubliniensis & 100 & 100 & 100 & 88.9 & 100 & 100 & 100 & 33.3 & 88.9 & 100 & 100 & 100 & 100 \\
\hline Candida glabrata & 100 & 88.9 & 88.9 & 88.9 & 100 & 100 & 77.8 & 33.3 & 77.8 & 100 & 100 & 100 & 100 \\
\hline Other yeast & 100 & 75 & 75 & 75 & 100 & 100 & 75 & 0 & 75 & 100 & 100 & 100 & 100 \\
\hline
\end{tabular}




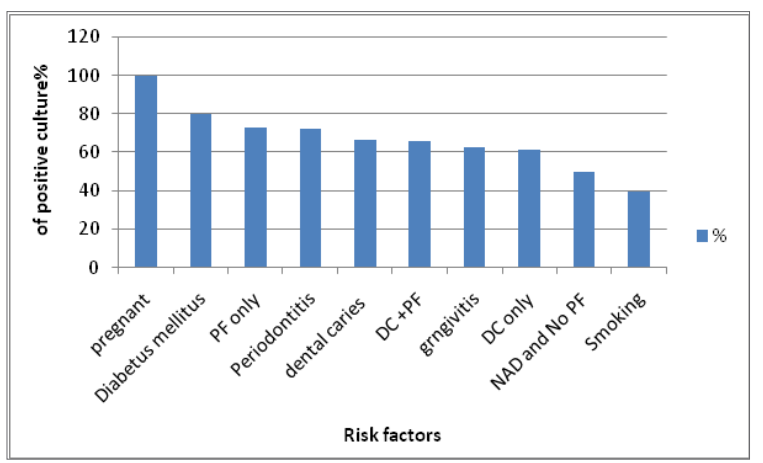

Figure I Yeast positive culture in patient with various risk factors with reference to positive

DC, dental complaint; PF, predisposing factors; NAD, no apparent disease.

\section{Discussion}

The present study to our knowledge is the first study to survey the prevalence of oral Candida species in Tripoli, Libya. The aim of this study was to investigate the carriage rate and background factors underlying carriage of oral Candida species in patient attending outpatient dental clinic in Tripoli district area. The carriage of Candida spp. in the oral cavities was $96(64 \%)$ over all. High oral carriage of yeast either in sex gender or age group was associated in all positive cultures with the presence of at least one dental compliment, in which 127 have at least one dental disease and $81(63.8 \%)$. was positive for yeast. In contrast to patient with no apparent disease (NAD), 23 and only 15 was culture positive for yeast (65.2\%), however 15 out 23 at least has one predisposing factor, this may explain high rate yeast carriage in NAD patient. Various study show that high oral carriage of candida in female than male, this may be attributed to the fact that female have more predisposing factors to be colonized and infected than male. ${ }^{15}$ Different study showed similar result indicates the importance role of dental compliant particular dental carriage gingivitis as well as Periodontitis in candida colonization. ${ }^{16}$ The rule of candida species especially candida albicans in oral cavity has been investigated and found to possess nanny virulence factors such as biofilm, adhesion and others to paly important rule in colonization and infection. ${ }^{17}$ This may explain its high frequent isolation in our study and others. ${ }^{18}$ Diabetes and pregnancy are the most important single Predisposing factor for yeast oral carriage in our study, this may also be related the fact that pregnancy and diabetes more prone and susceptible to candida colonization and infection not only oral site but also other such vaginitis in female. ${ }^{19}$ Our study also shows that most patient attending dental clinic with dental compliant as well as at least one predisposing factor. This may explain the high rate oral carriage by yeast and importance of both risk factors in oral colonization and infection. Considerable high carriage among our patients with no dental compliant and predisposing factor may be attributed to poor dental hygiene and cleaning. ${ }^{20}$

Isolation of C. glabrata and C. dubliniensis in our study as the most important second pathogen to be isolated from oral cavity was similar to other study. ${ }^{21,22}$ This finding can be related to the fact that both species has been emerged as an important pathogen in the last few years among immunocompromised patient and oral candidiasis either due antifungal resistant as with $C$. glabrata or virulence factors in C. Dubliniensis. ${ }^{23,24}$

All Candida isolates tested were susceptible to amphotericin B, nystatin, caspofungin, clotrimazole, miconazole and econazole. Furthermore, all C. albicans show high resistant to fluconazole, itraconazole, posoconazole and voriconazole. In contrast all other candida species including $C$. dubliniensis isolates were susceptible to most azole. However, C. glabrata isolates showed reduced susceptibility to fluconazole, itraconazole, posoconazole and voriconazole and some of these isolates were also resistant to ketoconazole. All candida species including other yeast were highly resistant to fluocytosine, this due innately resistant or rapid developed resistance. ${ }^{25}$ Resistance of C. albicans to fluconazole and other new azole possibly due to cross resistant between these classes of antifungal and have been reported by other studies as observed in our study. ${ }^{26}$ This is finding may guide our empiric treatment to shift for old azole in high-risk patients with known predisposing factors from developing serious candida infection particular with $C$. albicans. Identification of the infecting species such as C. glabrata or C. dubliniensis and other non-candida albicans could also be highly predictive of the likely drug susceptibility to azoles and could be used as a guide to therapy.

\section{Acknowledgements}

The authors acknowledged to thank medical staff of medical microbiology for their help and support during period of this study, particular Dr Arohma Amal and Saud Atloba.

\section{Conflict of interest}

Author declares that there is no conflict of interest.

\section{References}

1. Odds FC, Van Nuffel L, Dams G. Prevalence of Candida dubliniensis isolates in a yeast stock collection. J Clin Microbiol. 1998;36(10):28692873.

2. Saidi S, Luitaud C, Rouabhia M. In vitro synergistic effect of farnesol and human gingival cells against Candida albicans. Yeast. 2006;23(9):673687.

3. Siqueira JF, Sen BH. Fungi in endodontic infections. Oral Surg Oral Med Oral Pathol Oral Radiol Endod. 2004;97(5):632-641.

4. Loesche WJ, Schork A, Terpenning MS, et al. Factors which influence levels of selected organisms in saliva of older individuals. $J$ Clin Microbiol. 1995;33(10):2550-2557.

5. Thylstrup A. How should we manage initial and secondary caries? Quintessence Int. 1998;29(9):594-598.

6. Parolo CC, Maltz M. Microbial contamination of noncavitated caries lesions: a scanning electron microscopic study. Caries Res. 2006;40(6):536-541.

7. Sziegoleit F, Sziegoleit A, Wetzel WE. Effect of dental treatment and/or local application of amphotericin B to carious teeth on oral colonization by Candida. Med Mycol. 1999;37(5):345-350.

8. Tooyama H, Matsumoto $\mathrm{T}$, Hayashi, et al. Candida concentrations determined following concentrated oral rinse culture reflect clinical oral signs. BMC Oral Health. 2015;15:150.

9. Jan A, Bashir G, Fomda BA, et al. Hypertonic xylose agar medium: A novel medium for differentiation of Candida dubliniensis from Candida albicans. Indian J Med Microbiol. 2018;35(4):518-521.

10. Khan ZU, Ahmad S, Mokaddas E, et al. Simplified sunflower (Helianthus annuus) seed agar for differentiation of Candida dubliniensis from Candida albicans. Clin Microbiol Infect. 2004;10(6):590-592.

11. Nejad B, Rafiei A, Moosanejad F. Prevalence of Candida species in the oral cavity of patients with periodontitis. African Journal of Biotechnology. 2011;10(15):2987-2990.

12. CLSI. Methods for antifungal disk diffusion susceptibility testing of yeasts; approved standard M44-A. Clinical and Laboratory Standards Institute, Wayne, PA; 1997. 
13. CLSI. Method for antifungal disk diffusion susceptibility testing of yeasts; approved guideline. 2nd ed. CLSI document M44-A2. Wayne, PA: CLSI; 2009.

14.CLSI. Zone diameter interpretive standards, corresponding minimal inhibitory concentration (MIC) interpretive breakpoints, and quality control limits for antifungal disk diffusion susceptibility testing of yeasts. Third International Supplement CLSI document-M44-S3. Wayne, PA: CLSI; 2009.

15. de Resende MA, de Sousa LV, de Oliveira RC, et al. Prevalence and Antifungal Susceptibility of Yeasts Obtained from the Oral Cavity of Elderly Individuals. Mycopathologia. 2006;162(1):39-44.

16. de Carvalho FG, Silva DS, Hebling J, et al. Presence of mutans streptococci and Candida spp. in dental plaque/dentine of carious teeth and early childhood caries. Archives of Oral Biology. 2006;5(11):10241028.

17. Deepa K, Jeevitha T, Michael A. In vitro evaluation of virulence factors of Candida species isolated from oral cavity. Journal of Microbiology and antimicrobials. 2015;7(3):28-32.

18. Yang YL, Leaw SN, Wang AH, et al. Characterization of yeasts colonizing in healthy individuals. Med Mycol. 2011;49(1):103-106.

19. Mohammadi F, Javaheri MR, Nekoeian S, et al. Identification of Candida species in the oral cavity of diabetic patients. Curr Med Mycol. 2016;2(2):1-7.
20. Darwazeh AM, Al-Dwairi ZN, Al-Zwairi AA. The relationship between tobacco smoking and oral colonization with Candida species. J Contemp Dent Pract. 2010;11(3):17-24.

21. Li L, Redding S, Dongari-Bagtzoglou A. Candida glabrata, an Emerging Oral Opportunistic Pathogen. J Dent Res. 2007;86(3):204-215.

22. Marcos-Arias C, Vicente JL, Sahand IH, et al. Isolation of Candida dubliniensis in denture stomatitis. Archives of Oral Biology. 2009;54(2):127-131.

23. Collin B, Clancy CJ, Nguyen MH. Antifungal resistance in non- albicans Candida species. Drug Resistance Updates. 1999;2(1):9-14.

24. Vilela MM, Kamei K, Sano A, et al. Pathogenicity and virulence of Candida dubliniensis: comparison with C. albicans. Medical Mycology. 2002;40(3):249-257.

25. Law D, Moore CB, Wardle HM, et al. High prevalence of antifungal resistance in Candida spp. from patients with AIDS. J Anti Chemo. 1994;34(5):659-668.

26. Odds FC. Resistance of yeasts to azole-derivative antifungals. J Anti Chemo J Anti Chemo. 1993;31(4):463-471. 